BNL-112596-2016-JA

\title{
Effect of Precursor Selection on the Photocatalytic Performance of Indium Oxide Nanomaterials for Gas-Phase CO2 Reduction
}

\author{
Laura B. Hoch, Le He, Qiao Qiao, Kristine Liao, \\ Laura M. Reyes, Yimei Zhu, and Geoffrey A. Ozin
}

Submitted to CHEMISTRY OF MATERIALS

June 2016

Condensed Matter Physics and Material Science Department

Brookhaven National Laboratory

\author{
U.S. Department of Energy \\ USDOE Office of Science (SC), \\ Basic Energy Sciences (BES) (SC-22)
}

Notice: This manuscript has been authored by employees of Brookhaven Science Associates, LLC under Contract No. DE-SC0012704 with the U.S. Department of Energy. The publisher by accepting the manuscript for publication acknowledges that the United States Government retains a non-exclusive, paid-up, irrevocable, world-wide license to publish or reproduce the published form of this manuscript, or allow others to do so, for United States Government purposes. 


\section{DISCLAIMER}

This report was prepared as an account of work sponsored by an agency of the United States Government. Neither the United States Government nor any agency thereof, nor any of their employees, nor any of their contractors, subcontractors, or their employees, makes any warranty, express or implied, or assumes any legal liability or responsibility for the accuracy, completeness, or any third party's use or the results of such use of any information, apparatus, product, or process disclosed, or represents that its use would not infringe privately owned rights. Reference herein to any specific commercial product, process, or service by trade name, trademark, manufacturer, or otherwise, does not necessarily constitute or imply its endorsement, recommendation, or favoring by the United States Government or any agency thereof or its contractors or subcontractors. The views and opinions of authors expressed herein do not necessarily state or reflect those of the United States Government or any agency thereof. 


\title{
Effect of Precursor Selection on the Photocatalytic Performance of Indium Oxide Nanomaterials for Gas-Phase $\mathrm{CO}_{2}$ Reduction
}

\author{
Laura B. Hoch ${ }^{\dagger}$, Le He ${ }^{\dagger \ddagger}$, Qiao Qiao ${ }^{\S_{\delta}}$, Kristine Liao ${ }^{\dagger}$, Laura M. Reyes ${ }^{\dagger}$, Yimei Zhu ${ }^{\S}$, and Geoffrey \\ A. Ozin*† \\ ${ }^{\dagger}$ Department of Chemistry, University of Toronto, 8o St. George Street, Toronto, Ontario $\mathrm{M}_{5} \mathrm{~S} 3 \mathrm{H} 6$, Canada. ${ }^{\ddagger}$ Pre- \\ sent Address: Institute of Functional Nano \& Soft Materials (FUNSOM), Jiangsu Key Laboratory for Carbon-Based \\ Functional Materials \& Devices, Soochow University, 199 Ren'ai Road, Suzhou, 215123, Jiangsu, PR China. ${ }^{\S}$ Con- \\ densed Matter Physics and Materials Science Department, Brookhaven National Laboratory, Upton, NY, 11973, USA. \\ ${ }^{\delta}$ Department of Physics, Temple University, Philadelphia, PA, 19122, USA. \\ * Corresponding author. Email: gozin@chem.utoronto.ca
}

KEYWORDS Solar Fuels, Indium Oxide, Photocatalysis, Carbon Dioxide, Surface Defects

\begin{abstract}
Highly defected indium oxide nanoparticles, $\operatorname{In}_{2} \mathrm{O}_{3-\mathrm{x}}(\mathrm{OH})_{\mathrm{y}}$, have been shown to function as active photocatalysts for gas-phase $\mathrm{CO}_{2}$ reduction under simulated solar irradiation. Herein we demonstrate that the choice of starting material has a strong effect on the photocatalytic activity of indium oxide nanoparticles. We examine three indium oxide materials prepared via the thermal decomposition of either indium (III) hydroxide or indium (III) nitrate and correlate their stability and photocatalytic activity to the number and type of defect present in the material. Further, we use ${ }^{13} \mathrm{CO}_{2}$ isotope-tracing experiments to clearly identify the origins of the observed carbon-containing products. Significantly, we find that the oxidizing nature of the precursor anion has a substantial impact on the defect formation within the sample. This study demonstrates the importance of surface defects in designing an active heterogeneous photocatalyst and provides valuable insight into key parameters for the precursor design, selection and performance optimization of materials for gas-phase $\mathrm{CO}_{2}$ reduction.
\end{abstract}

Harnessing abundant solar energy to drive the conversion of greenhouse gas $\mathrm{CO}_{2}$ into carbon-based fuels and chemical feedstocks - a process analogous to nature's photosynthesis - is an area of growing scientific focus, with the ultimate goal of providing a sustainable alternative to traditional fossil-based carbon. ${ }^{1-4}$ Enabling this technology would allow the capture and conversion of $\mathrm{CO}_{2}$ emitted from energy production and manufacturing exhaust streams into valuable products, thereby creating significant economic and environmental benefits. ${ }^{5-7}$ Of particular interest is the development of gas-phase heterogeneous photocatalysts based on semiconductor nanomaterirals, which can efficiently harvest incident solar energy, utilizing it to drive useful molecular transformations at its surface. ${ }^{3,8,9}$ Nanostructured metal oxides have shown significant promise as photocatalysts for $\mathrm{CO}_{2}$ reduction, due to their stability, ease of synthesis and scale-up. $^{10-12}$

The role of bulk and surface defects in determining the photocatalytic activity of metal oxide semiconductor photocatalysts has been an area of intense research. ${ }^{13-16}$ In metal oxides, perhaps the most ubiquitous and wellstudied type of defect is the oxygen vacancy. ${ }^{17-19}$ These oxygen vacancies have been shown to have a significant impact on the optical and electronic properties. ${ }^{13,20}$ Typically acting as donor states, they are generally cited as the primary reason for the n-type conductivity associated with most metal oxides. ${ }^{21-23}$ Oxygen vacancies can also act as important active sites in heterogeneous catalysis reactions, facilitating reactant adsorption and conversion. ${ }^{7,24}$ For example, in $\mathrm{TiO}_{2}$, it has been suggested that surface oxygen vacancies act as charge traps, facilitating electron transfer to adsorbed species, while bulk oxygen vacancies act as recombination centers, lowering the excited state charge carrier lifetimes and adversely affecting the photocatalytic activity. ${ }^{25}$ Surface hydroxide groups have also been shown to play a key role in photocatalysis reactions. ${ }^{13,24,26,27}$ In many materials, they have commonly been associated with facilitating the adsorption of gaseous reactant species. Indeed, surface hydroxides have a known affinity for $\mathrm{CO}_{2}$, typically forming surface adsorbed carbonate or bicarbonate species. ${ }^{28}$ Several groups have reported positive correlations with hydroxide content, $\mathrm{CO}_{2}$ capture capacity, and enhanced photocatalytic activity. ${ }^{24,26,27}$ 
In our previous work, we demonstrated that defected indium oxide nanoparticles, $\operatorname{In}_{2} \mathrm{O}_{3-\mathrm{x}}(\mathrm{OH})_{\mathrm{y}}$, function as active photocatalysts for $\mathrm{CO}_{2}$ reduction to $\mathrm{CO}$ via the reverse water gas shift (RWGS) reaction. ${ }^{29}$ We correlated both the photocatalytic activity and $\mathrm{CO}_{2}$ capture capacity to the oxygen vacancy and surface hydroxide content of the $\operatorname{In}_{2} \mathrm{O}_{3-\mathrm{x}}(\mathrm{OH})_{\mathrm{y}}$ samples, suggesting both defects play a key role in the reaction mechanism..$^{30,31}$ These samples were produced via thermal dehydroxylation of indium (III) hydroxide. During this reaction, adjacent hydroxide groups condense to form a bridging oxide and release water. We demonstrated that the calcination temperature during the synthesis of these nanoparticles had a strong effect on the amount of hydroxide groups retained within the sample: lower temperatures resulted in less condensation and formation of bridging oxide bonds, resulting in the retention of more hydroxides and oxygen vacancy defects. We observed a strong positive correlation between $\mathrm{CO}_{2}$ capture capacity and hydroxide content. However, an $\operatorname{In}(\mathrm{OH})_{3}$ control sample, which had the highest hydroxide content, showed a significantly lower $\mathrm{CO}_{2}$ capture capacity, even when corrected for surface area. This indicated that surface hydroxides alone are not sufficient to facilitate $\mathrm{CO}_{2}$ capture and subsequent photocatalytic reduction of $\mathrm{CO}_{2}$. However, due to the temperaturedependence of the synthesis, which affects the retention of both hydroxides and oxygen vacancies, it is difficult to separate and evaluate the effects these two defects have on the reactivity of the different samples.

In order to gain better insight into the reaction mechanism and understand the role hydroxides and oxygen vacancies play in the formation of an active indium oxide based photocatalyst, we sought to make indium oxide from a non-hydroxide containing precursor. Herein we examine the photocatalytic activity of three different indium oxide samples prepared via the thermal decomposition of either indium (III) hydroxide or indium (III) nitrate. Our results demonstrate that the choice of starting material has a significant effect on the number and type of defects present in the material. In particular, we find that the oxidizing nature of the precursor anion has a substantial impact on the defect formation within the sample, which strongly correlates with the stability and photocatalytic activity. This work significantly advances our understanding of the role precursor anions play in determining a material's photocatalytic activity and further illustrates the importance of surface defects in designing an active material capable of facilitating gas-phase $\mathrm{CO}_{2}$ reduction.

\section{Experimental}

\section{Synthesis of indium oxide nanoparticles}

All chemicals were used as received without any further purification. The indium (III) hydroxide precursor was prepared by dissolving indium(III) chloride (3.6 g, 16.2 mmol, Sigma Aldrich, 98\%) in a 3:1 solution (72 mL) of anhydrous ethanol (Commercial Alcohols) and deionized, nanopure water (resistivity 18.2 $\mathrm{M} \Omega \mathrm{cm}$ ). In a separate beaker, a 3:1 mixture of ethanol and ammonium hydroxide was prepared by combining aqueous ammonium hy- droxide (18 mL, Caledon, $28-30 \%$ adjusted to $25 \mathrm{wt} \%$ with deionized water) with anhydrous ethanol $(54 \mathrm{~mL})$. The solutions were rapidly combined, resulting in the immediate formation of a white precipitate. To control the particle size, the resulting suspension was immediately immersed in a pre-heated oil bath at $80{ }^{\circ} \mathrm{C}$ and stirred for 10 min. The suspension was then removed from the oil bath and allowed to cool to room temperature. The precipitate was separated via centrifugation and washed 3 times with deionized water. The precipitate was sonicated between washings to ensure adequate removal of any trapped impurities and then dried overnight at $80{ }^{\circ} \mathrm{C}$ in a vacuum oven. The dried precursor powder was finely ground with a mortar and pestle.

Each of the indium oxide samples were made by calcining $0.8 \mathrm{~g}$ of the respective precursor - either indium (III) hydroxide (prepared as described above), indium (III) nitrate hydrate (Sigma Aldrich, 99.9\%), indium (III) acetate (anhydrous, Acros Organics, 99.99\%), or indium (III) chloride (anhydrous, Alfa Aesar, 99.99\%) at $250{ }^{\circ} \mathrm{C}$ for 3 hrs. The resulting powders were finely ground with a mortar and pestle.

High surface area indium oxide made from the indium (III) nitrate precursor was prepared via a $\mathrm{SiO}_{2}$-templating method, illustrated in Figure $\mathrm{S}_{3}$. Indium (III) nitrate hydrate (3 g, Sigma Aldrich, 99.9\%) was dissolved in $30 \mathrm{~g}$ of Ludox HS-40 colloidal silica nanoparticles (Aldrich, 40 $w t \%$ in water) and sonicated for 30 min to ensure homogeneity. The solution was dried overnight at $80^{\circ} \mathrm{C}$ and the resulting solid was directly calcined at $25^{\circ}{ }^{\circ} \mathrm{C}$ for $3 \mathrm{hrs}$. After calcination the solid changed from white to pale yellow indicating the formation of indium oxide. This $\mathrm{In}_{2} \mathrm{O}_{3} / \mathrm{SiO}_{2}$ mixture was then ground with an agate mortar and pestle and the $\mathrm{SiO}_{2}$ was removed by base etching. Specifically, the $\mathrm{In}_{2} \mathrm{O}_{3} / \mathrm{SiO}_{2}$ powder sample was dispersed in $2 \mathrm{M} \mathrm{NaOH}$ (10x excess by mass) and sonicated overnight (approximately 20 hrs). The suspension was then heated to $80{ }^{\circ} \mathrm{C}$ with a high stirring rate and heated for an additional $24 \mathrm{hrs}$. The resulting sample was separated via centrifugation and purified by washing five times with deionized nanopure water, sonicating between washings to ensure adequate removal of any trapped impurities. The final precipitate was dried overnight at $70{ }^{\circ} \mathrm{C}$ in a vacuum oven and finely ground with a mortar and pestle. Several control samples were also prepared. The indium nitrate was replaced with the indium hydroxide precursor ( $0.6 \mathrm{~g})$, combined with $10 \mathrm{~g}$ of Ludox HS-40, sonicated, dried, calcined, etched, and purified in exactly the same way as described above. Additionally, two indium oxide samples (0.5 g, prepared without $\mathrm{SiO}_{2}$ from standard indium nitrate and indium hydroxide precursors as described in the previous paragraph) were placed in $50 \mathrm{ml}$ of $2 \mathrm{M} \mathrm{NaOH}$, exposed to the same basic etching conditions and purified in the same way as described above.

Sample films were prepared for photocatalytic testing by drop casting $20 \mathrm{mg}$ of each sample powder - suspended via sonication in deionized, nanopure water $(3 \mathrm{~mL})$ - onto 1"x1" binder free borosilicate glass microfiber filters (Whatman, GF/F, o.7 $\mu \mathrm{m}$ ). 

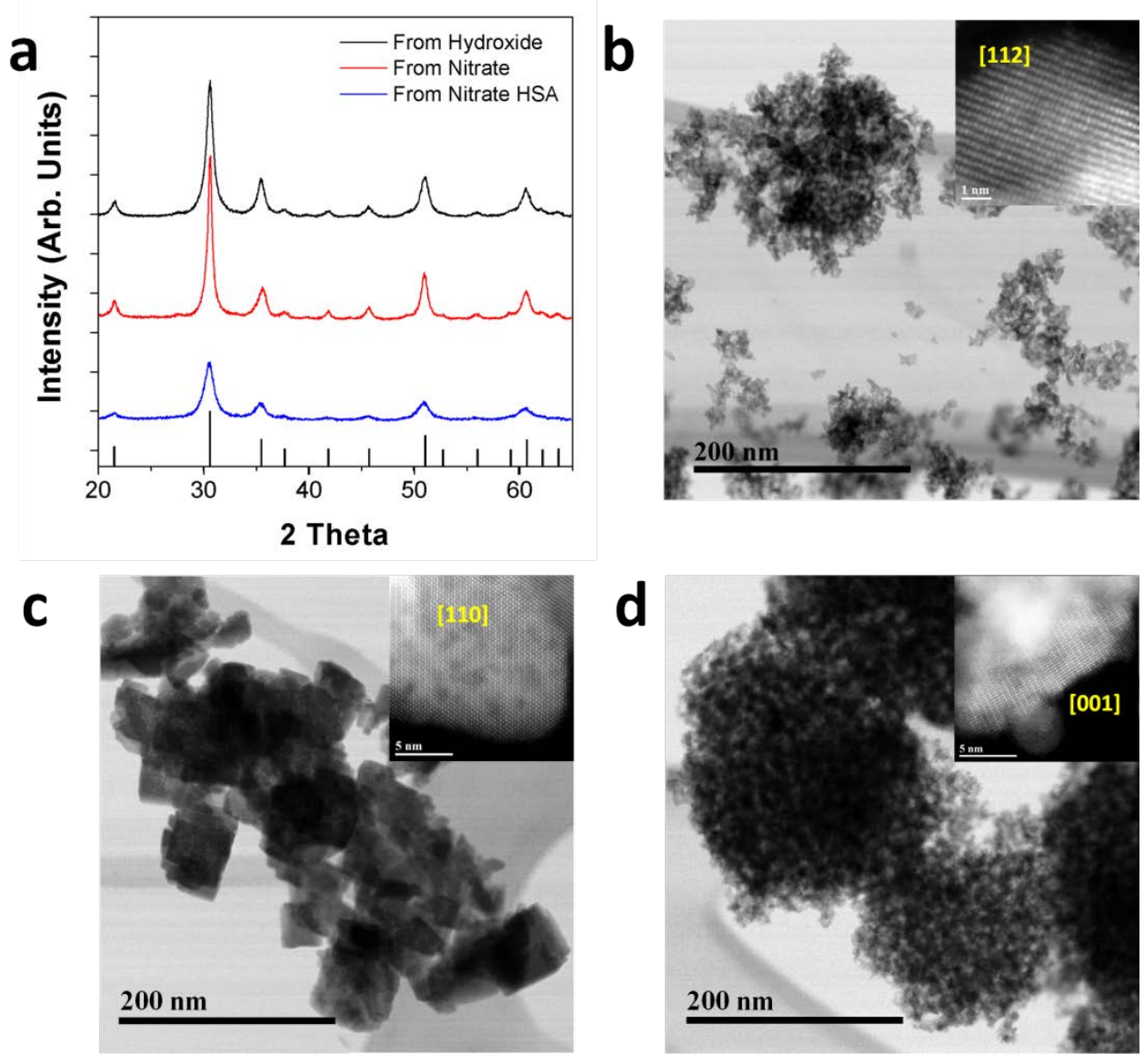

Figure 1. (a) Powder X-ray diffraction patterns for samples made from different indium (III) precursors. (b-d) Scanning transmission electron microscope (STEM) images of (b) $\mathrm{In}_{2} \mathrm{O}_{3}$ made from indium (III) hydroxide, (c) $\operatorname{In}_{2} \mathrm{O}_{3}$ made from indium (III) nitrate, and (d) high surface area (HSA) $\mathrm{In}_{2} \mathrm{O}_{3}$ sample prepared from indium (III) nitrate via the $\mathrm{SiO}_{2}$ templating method.

\section{Physical Characterization}

Powder X-ray diffraction (PXRD) was performed on a Bruker D2-Phaser X-ray diffractometer, using $\mathrm{Cu} \mathrm{K} \alpha$ radiation at $30 \mathrm{kV}$. Nitrogen adsorption isotherms were obtained at $77 \mathrm{~K}$ using a Quantachrome Autosorb-1-C, and the surface area was determined using Brunauer-EmmetTeller (BET) theory. Sample morphology was determined using an aberration corrected JEOL JEM-ARM2ooCF scanning transmission electron microscope (STEM). The atomic resolution high angle annular dark field (HAADF) and low magnification bright field images were taken at $200 \mathrm{kV}$. The diffuse reflectance of the samples was measured using a Perkin Elmer Lambda 1050 UV/VIS/NIR spectrometer equipped with an integrating sphere with a diameter of $150 \mathrm{~mm}$.

X-ray photoelectron spectroscopy (XPS) was performed using a Perkin Elmer Phi 5500 ESCA spectrometer in an ultrahigh vacuum chamber with base pressure of $1 \times 10^{-9}$ Torr. The spectrometer uses an $\mathrm{Al} \mathrm{K} \alpha$ X-ray source operating at $15 \mathrm{kV}$ and $27 \mathrm{~A}$. The samples used in XPS analyses were prepared by drop-casting aqueous dispersions onto p-doped $\mathrm{Si}(100)$ wafers. All data analyses were carried out using the Multipak fitting program and the binding ener- gies were referenced to the NIST-XPS database ${ }^{32}$ and the Handbook of X-ray Photoelectron Spectroscopy. ${ }^{38}$

\section{Gas-phase Photocatalytic Measurements}

Gas-phase photocatalytic rate measurements were conducted in a custom fabricated $1.5 \mathrm{~mL}$ stainless steel batch reactor with a fused silica view port sealed with Viton Orings. Briefly, the reactors were evacuated using an Alcatel dry pump prior to being purged with $\mathrm{H}_{2}$ gas (99.9995\%) at a flow rate of $6 \mathrm{~mL} / \mathrm{min}$ for 1 hour. The outlet valve was sealed and the reactors were injected with $1 \mathrm{~atm}$ of ${ }^{13} \mathrm{CO}_{2}$ (99.9 atomic \% Sigma Aldrich). The inlet valve was then sealed and the reactors were heated to $150^{\circ} \mathrm{C}$. The reactor temperatures were controlled by an OMEGA CN616 6Zone temperature controller, with a thermocouple placed in contact with the sample. The pressure inside the reactor was monitored during the reaction using an Omega $\mathrm{PX}_{309}$ pressure transducer. Reactors were irradiated with a 1000 W Hortilux Blue metal halide bulb for a period of 16 hours. Product gases were analyzed by a flame ionization detector (FID) and thermal conductivity detector (TCD) installed in a SRI-8610 Gas Chromatograph (GC) with a 3' Mole Sieve 13a and 6' Haysep D column. Isotope product gases were measured using an Agilent $7890 \mathrm{~A}$ gas 
chromatographic mass spectrometer (GC-MS) with a 6o $\mathrm{m}$ GS-Carbonplot column fed to the mass spectrometer.

\section{Results and Discussion}

\section{Preparation of indium oxide nanoparticles from differ- ent precursors}

In order to better understand the role hydroxides play in the formation of an active $\operatorname{In}_{2} \mathrm{O}_{3}$-based photocatalyst, we sought to make indium oxide from a non-hydroxide containing precursor. To this end, we calcined four indium (III) precursor salts with different anions (nitrate, acetate, chloride, and hydroxide) at $250{ }^{\circ} \mathrm{C}$ for 3 hrs, conditions which our previous studies have demonstrated are optimal for producing the most active indium oxide photocatalysts. ${ }^{29}$ Of the four indium (III) precursor salts, only indium (III) hydroxide and indium (III) nitrate produced pure crystalline indium oxide with no observable amorphous phases, as shown in the powder X-ray diffraction (PXRD) patterns in Figure 1a. The indium (III) chloride and indium (III) acetate precursors on the other hand did not produce pure indium oxide as shown in Figures $\mathrm{S} 1$ and $S_{2}$ in the supporting information.

Figures $\mathrm{ib}$ and $1 \mathrm{c}$ show the STEM images of the indium oxide samples produced from indium (III) hydroxide and indium (III) nitrate, respectively. From the images it is clear that the morphology differs significantly between the samples. The hydroxide-based $\operatorname{In}_{2} \mathrm{O}_{3}$ sample (Figure $\mathrm{lb}$ ) is comprised of small agglomerated nanoparticles with diameters less than $10 \mathrm{~nm}$. On the other hand, the nitratebased $\mathrm{In}_{2} \mathrm{O}_{3}$ sample (Figure 1c) exhibits larger, more faceted polydisperse nanoparticles in the range of $20-50 \mathrm{~nm}$. From these images, it is evident that the nitrate-based $\mathrm{In}_{2} \mathrm{O}_{3}$ sample has a much lower surface area than the hydroxide-based sample. To quantify this, we measured their BET surface areas and found that there was a stark difference: $3 \mathrm{~m}^{2} / \mathrm{g}$ for the nitrate-based sample and 125 $\mathrm{m}^{2} / \mathrm{g}$ for the hydroxide-based sample. Because the photoreduction of $\mathrm{CO}_{2}$ on indium oxide is a surface-mediated reaction, the substantially lower surface area of the nitrate-based sample could result in significantly reduced apparent photocatalytic activity.

In order to be able to more directly compare the nitrate- and hydroxide-based samples, we sought to synthesize a higher surface area nitrate-based $\operatorname{In}_{2} \mathrm{O}_{3}$ sample. As was demonstrated in previous work, the calcination temperature has a significant impact on the catalytic activity, therefore maintaining the calcination time and temperature is important to ensure consistency between the samples. Because $\mathrm{In}_{2} \mathrm{O}_{3}$ is generally considered to be very stable under basic conditions, ${ }^{33}$ we chose to use $\mathrm{SiO}_{2}$ spheres as an inert template, which could then be etched away using a strong base. In this process, illustrated schematically in Figure $\mathrm{S}_{3}$, the indium (III) nitrate precursor is dissolved in an aqueous suspension of $\mathrm{SiO}_{2}$ nanoparticles and allowed to dry slowly overnight, encapsulating the $\mathrm{SiO}_{2}$ nanoparticles within the indium nitrate salt. Once dry, the sample is then calcined for 3 hours at $250{ }^{\circ} \mathrm{C}$ to convert the indium nitrate into $\operatorname{In}_{2} \mathrm{O}_{3}$. To remove the

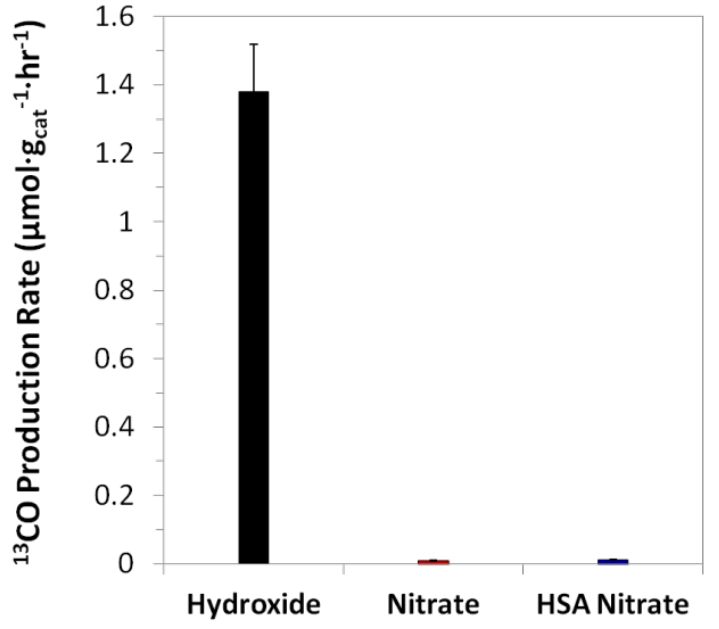

Figure 2. Photocatalytic performance of the three indium oxide samples made from different precursors under 800 $\mathrm{W} \mathrm{m}{ }^{-2}$ (o.8 suns) illumination at $150{ }^{\circ} \mathrm{C}$. All values for carbon monoxide were confirmed using ${ }^{13} \mathrm{C}$ isotope tracing.

$\mathrm{SiO}_{2}$, the sample is mechanically ground into a fine powder and then dispersed in a strongly basic solution. In order to reduce particle aggregation and ensure as much of the $\mathrm{SiO}_{2}$ is exposed to the basic solution as possible, this suspension was sonicated overnight prior to heating at $80{ }^{\circ} \mathrm{C}$ for 24 hours to complete the etching procedure. The resulting fine powder was washed extensively with deionized water to remove impurities

Figure id shows an STEM image of the high surface area (HSA) nitrate-based sample prepared using this method. The highly porous structure is evident from this image and gas adsorption measurements indicate that the BET surface area of the nitrate sample was successfully increased from $3 \mathrm{~m}^{2} / \mathrm{g}$ to $181 \mathrm{~m}^{2} / \mathrm{g}$. The PXRD patterns in Figure 1a show the comparison of the HSA-nitrate-based sample with the original nitrate-based sample and the hydroxide-based sample. As the PXRD pattern indicates, the HSA-nitrate sample is comprised of pure crystalline $\mathrm{In}_{2} \mathrm{O}_{3}$. No evidence of any crystalline side phases or residual amorphous $\mathrm{SiO}_{2}$ is observed. The peaks of the HSAnitrate sample show significant broadening relative to the nitrate- and hydroxide-based samples, indicating a decrease in particle size, which is consistent with the TEM data.

\section{Investigating the effects of precursor choice on photo- catalytic activity}

Figure 2 shows the photocatalytic activity of the three $\mathrm{In}_{2} \mathrm{O}_{3}$ samples under $800 \mathrm{~W} \cdot \mathrm{m}^{-2}$ (o.8 suns) illumination at $150{ }^{\circ} \mathrm{C}$ under 2 atm of ${ }^{13} \mathrm{C}$-labelled ${ }^{13} \mathrm{CO}_{2}$ and $\mathrm{H}_{2}$ in a 1:1 ratio, which is stoichiometric for the reverse water gas-shift reaction. ${ }^{13} \mathrm{C}$-labelling is essential in order to prove that the source of the carbon containing products originates from the reactant $\mathrm{CO}_{2}$ and not from carbon contamination on the catalyst's surface or in the reactor. Of the three samples, only the $\operatorname{In}_{2} \mathrm{O}_{3}$ made from the hydroxidebased precursor showed activity for ${ }^{13} \mathrm{CO}_{2}$ reduction, 

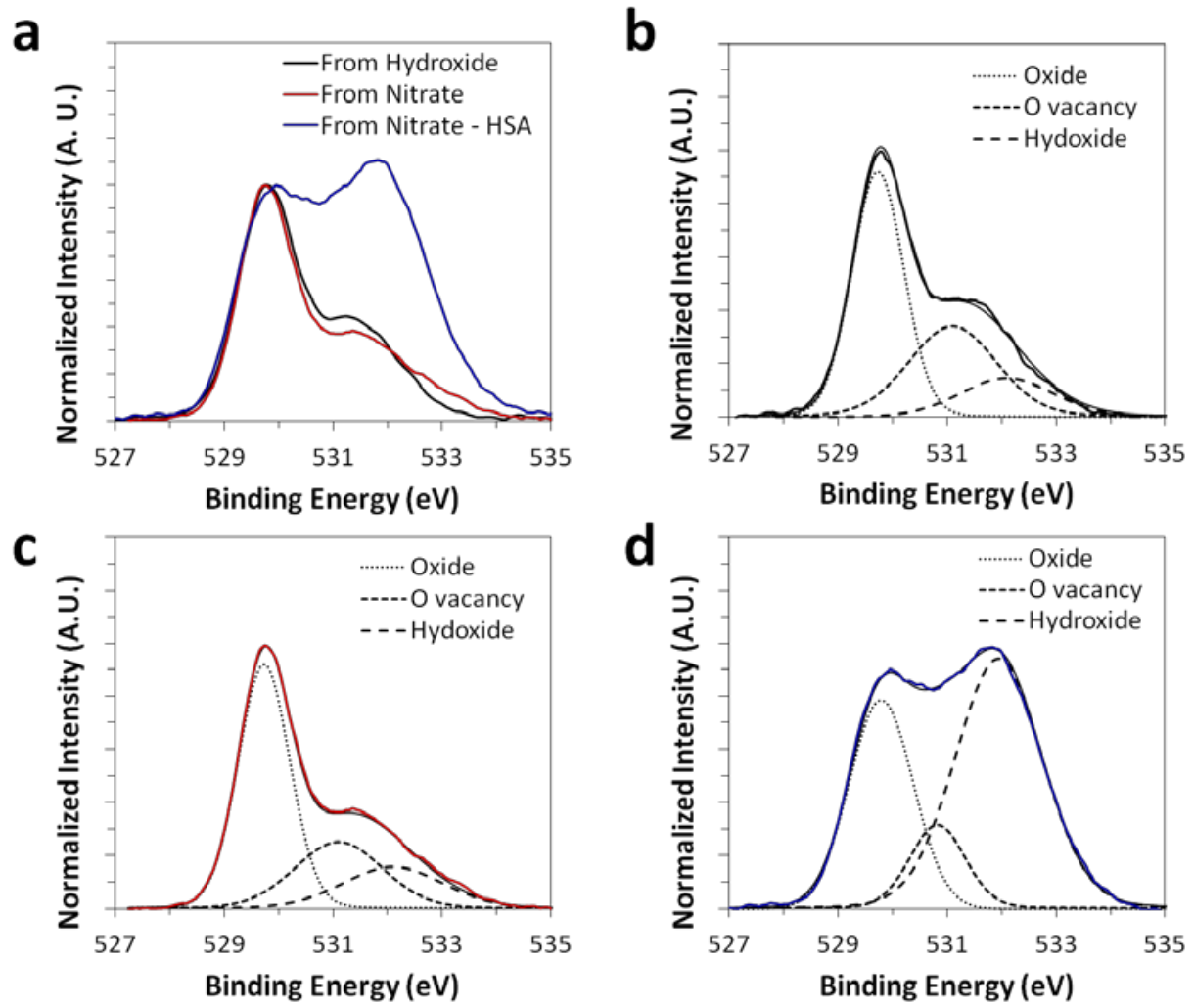

Figure 3. Comparison of XPS spectra for $\operatorname{In}_{2} \mathrm{O}_{3}$ samples prepared from either indium hydroxide or indium nitrate precursors. (a) Overlay of the O1s peaks for all samples. Qualitative de-convolution of the O1s peaks for $\operatorname{In}_{2} \mathrm{O}_{3}$ prepared from (b) indium (III) hydroxide, (c) indium (III) nitrate, and (d) high surface area indium (III) nitrate. The main peak at $529.9 \mathrm{eV}$ is attributed to indium oxide. The two additional shoulder peaks at $531.0 \mathrm{eV}$ and $532.1 \mathrm{eV}$ are attributed to oxygen vacancies in the structure and surface $\mathrm{OH}$ groups, respectively.

producing pure ${ }^{13} \mathrm{C}$-labelled $\mathrm{CO}$ at a rate of $1.38 \mu \mathrm{mol} \cdot \mathrm{g}_{\text {cat }}$ ${ }^{1} \cdot \mathrm{hr}^{-1}$ with no evidence of any other $\mathrm{C}$-containing products. This indicates that ${ }^{13} \mathrm{CO}$ is the sole product of $\mathrm{CO}_{2}$ reduction, originating from the reactant ${ }^{13} \mathrm{CO}_{2}$ gas and not from adventitious carbon contamination. For reference, this reaction rate is consistent with ${ }^{13} \mathrm{CO}$ production rates reported for other single component metal oxide materials under similar conditions; for instance $1.61 \mu \mathrm{mol} \cdot \mathrm{g}_{\text {cat }}{ }^{-1} \cdot$ hour $^{-1}$ for $\mathrm{MgO},{ }^{34}$ and $0.56 \mu \mathrm{mol} \cdot \mathrm{g}_{\text {cat }}{ }^{-1} \cdot$ hour $^{-1}$ for $\mathrm{ZrO}_{2}{ }^{35}$

Conversely, both nitrate-based $\operatorname{In}_{2} \mathrm{O}_{3}$ samples did not produce ${ }^{13} \mathrm{CO}$ above the baseline level, nor were any other $\mathrm{C}$-containing products observed. Because both the original nitrate-based sample and the HSA-nitrate-based $\operatorname{In}_{2} \mathrm{O}_{3}$ sample show no evidence of photocatalytic activity, this demonstrates that surface area alone is not responsible for the observed absence of activity for the nitrate-based samples.

\section{Characterization of defect formation as a function of $\ln _{2} \mathrm{O}_{3}$ precursor}

In order to better understand why the nitrate-based samples are inactive towards $\mathrm{CO}_{2}$ reduction, X-ray photoelectron spectroscopy (XPS) measurements were performed to characterize the defects present in the $\operatorname{In}_{2} \mathrm{O}_{3}$ samples, as well as confirm the sample composition to determine if there are any impurities which might be adversely impacting the photocatalytic activity. Figure $\mathrm{S}_{4}$ shows the survey spectra for all three samples, which are remarkably similar. No nitrogen signal is observed in either of the nitrate-based samples (Figure $\mathrm{S}_{4} \mathrm{~b}$ and c). The $\mathrm{N}$ is peak should appear at a binding energy between 397$408 \mathrm{eV},{ }^{32}$ however there is no evidence of any signal in this region of the spectrum. This indicates that nitrogen is sufficiently removed upon calcination of the indium nitrate and should not affect the photocatalytic performance of the nitrate-based samples. The only obvious difference in these spectra is the appearance of $\mathrm{Si} 2 \mathrm{~s}$ and 2p peaks at $157 \mathrm{ev}$ and $105 \mathrm{eV}$, respectively, in the HSAnitrate-based sample (Figure $\mathrm{S}_{4 \mathrm{C}}$ ). This is likely due to residual $\mathrm{SiO}_{2}$ nanoparticles that were completely encapsulated by $\operatorname{In}_{2} \mathrm{O}_{3}$ and therefore inaccessible to the $\mathrm{NaOH}$ etching solution. However, $\mathrm{SiO}_{2}$ is a commonly used inert support material and it is unlikely that residual $\mathrm{SiO}_{2}$ nanoparticles had a negative impact on the photocatalytic activity of the HSA-nitrate-based samples. ${ }^{36}$ Based on these results, we do not expect that atomic impurities are responsible for the difference in reactivity between the hydroxide-based and nitrate-based $\operatorname{In}_{2} \mathrm{O}_{3}$ samples. 
From Hydroxide
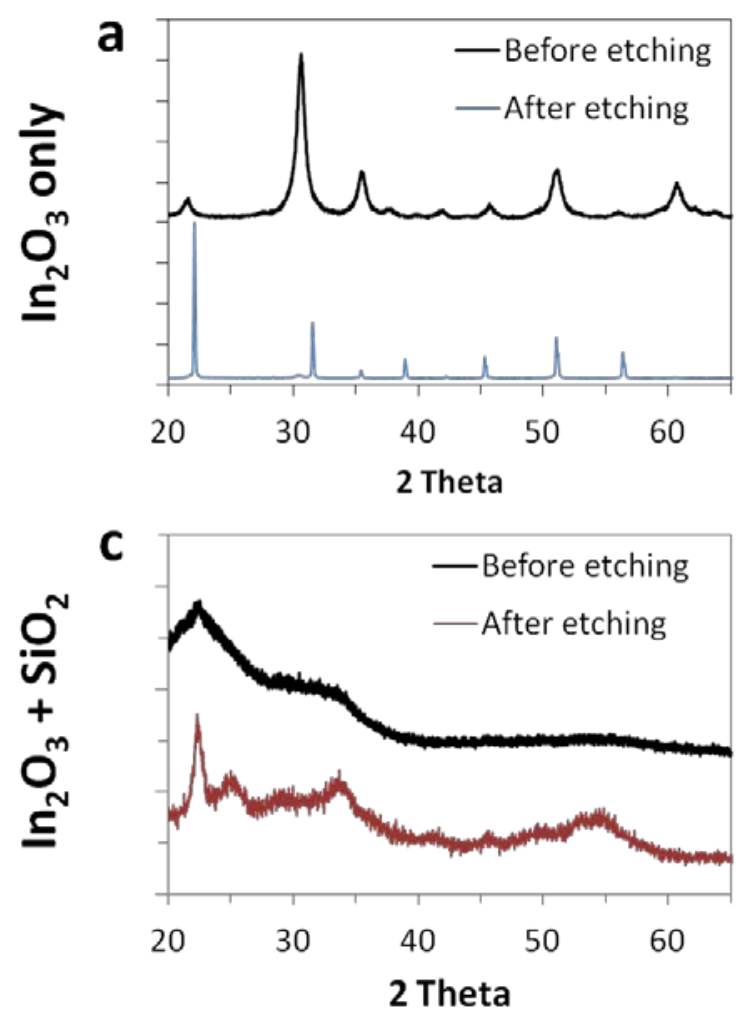

From Nitrate

b

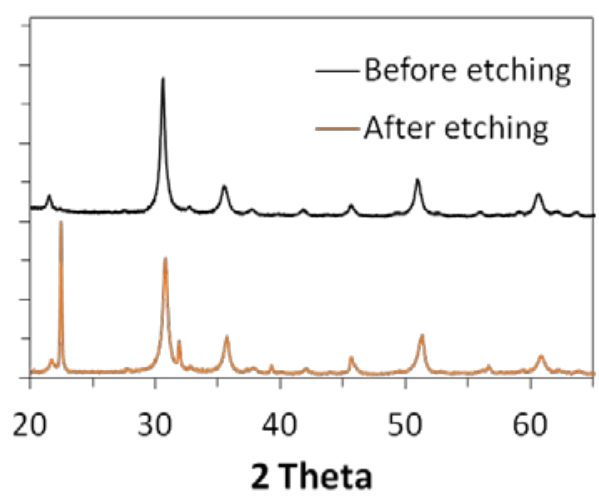

d

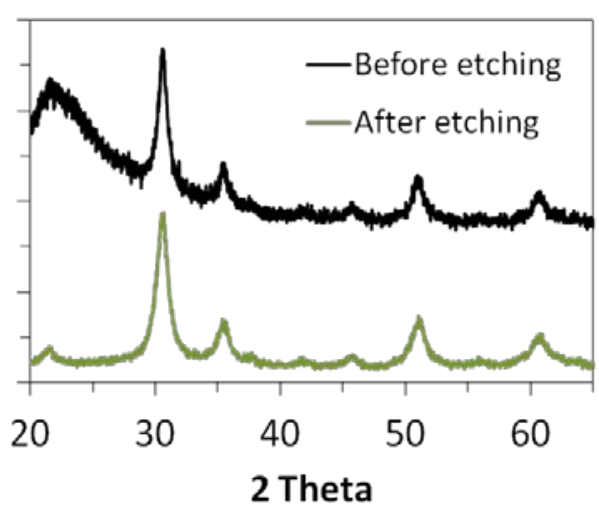

Figure 4. Powder x-ray diffraction patterns before and after base treatment of calcined powder samples containing: $(a) \mathrm{In}_{2} \mathrm{O}_{3}$ only - from indium hydroxide, (b) $\mathrm{In}_{2} \mathrm{O}_{3}$ only - from indium nitrate, (c) $\operatorname{In}_{2} \mathrm{O}_{3}+\mathrm{SiO}_{2}-$ from indium hydroxide, and (d) ) In $\mathrm{O}_{3}+$ $\mathrm{SiO}_{2}$ - from indium nitrate. The y-axis of all four graphs represents intensity in arbitrary units.

Another possible reason for the difference in activity between the samples could be related to the type and quantity of defects within the $\mathrm{In}_{2} \mathrm{O}_{3}$ itself. In our previous work, we demonstrated the effect of calcination temperature on the photocatalytic activity of the $\mathrm{In}_{2} \mathrm{O}_{3}$ samples and the strong correlation of activity to both oxygen vacancies and surface hydroxides. ${ }^{29}$ As the calcination temperature was increased, the quantity of both hydroxide and oxygen vacancy defects decreased, as did the photocatalytic activity. In this study, all samples were calcined at the same temperature, $250{ }^{\circ} \mathrm{C}$; however as indicated by the O1s core level peaks shown in Figure 3, there is a substantial difference between the three $\operatorname{In}_{2} \mathrm{O}_{3}$ samples. The shoulder peak that appears at higher binding energies indicates that there is more than one chemical state of oxygen present in the structure. Indeed, the O1s peak can be de-convoluted into three distinct peaks: the main oxide peak at $529.9 \mathrm{eV}$, and two additional peaks at $531.0 \mathrm{eV}$ and 532.1 eV (Figure 3b-d). The peak at 531.0 eV is commonly attributed to the presence of oxygen vacancies in the structure. ${ }^{37,38}$ The shift to higher binding energy for this oxygen vacancy peak relative to the main oxide peak is a result of the change in oxygen atom interaction with indium centers that are more reduced in character because they are surrounded by less than 6 oxygen atoms (due to the oxygen vacancies). The peak at $532.1 \mathrm{eV}$ is generally attributed to surface $\mathrm{OH}$ groups. ${ }^{37}$ From these plots, one can obtain a qualitative understanding of the relative differences in defect type and quanitity in the three $\mathrm{In}_{2} \mathrm{O}_{3}$ samples. As shown in Figure 3a, the regular nitrate-based and the hydroxide-based samples have shoulder peaks with similar intensities, however the primary difference between them is their shape. The nitratebased sample has a higher signal at higher binding energies, indicating a greater contribution from hydroxides (Figure 3c), while the hydroxide-based sample has a higher intensity at intermediate binding energies, indicating a greater contribution from oxygen vacancies (Figure $3 \mathrm{~b}$ ). Indeed the hydroxide-based sample has the highest contribution from oxygen vacancies of all three samples that were measured. The HSA-nitrate-based sample has the highest shoulder peak overall, and has a substantial contribution in the hydroxide region (Figure $3 \mathrm{~d}$ ), which is not surprising given that this sample was prepared by etching in strong base for two days. It should be noted that $\mathrm{Si}-\mathrm{O}$ bonds arising from the encapsulated $\mathrm{SiO}_{2}$ nanoparticles may also contribute to the O1s shoulder peak. However, as shown in the XPS survey spectrum (Figure S4c), the Si signal is very small relative to the In and $\mathrm{O}$ signals, so the contribution of $\mathrm{Si}-\mathrm{O}$ bonds to the O1s peak would likely also be very small. Further, the signal arising from $\mathrm{Si}-\mathrm{O}$ bonds tends to be at higher binding energies ( -532.5-533.0 $\mathrm{eV}$ ) than what we observed for the shoulder peak, which indicates that while there may be some contribution from 
Si-O bonds, it is not likely the dominant source of the O1s shoulder peak.

These results, comparing the different defect populations in the hydroxide-based and nitrate-based samples, indicate that the lower oxygen vacancy content in the nitrate-based sample relative to the hydroxide-based sample could be responsible for the observed difference in photocatalytic activity. Indeed, many studies have demonstrated that an increase in oxygen vacancy content is strongly correlated to an increase in photocatalytic activity in metal oxide materials. ${ }^{13,39}$ Even in relatively small quantities, oxygen vacancies can play a key role in determining the activity of heterogeneous catalysts by not only acting as active sites for the reaction to take place, but also alter the electronic properties of the material. ${ }^{21,40,41}$ Therefore, although the difference in oxygen vacancies population is small, it could explain why the hydroxidebased sample has a much higher photocatalytic activity.

Because the population of defects clearly has a strong impact on photocatalytic activity of indium oxide, in order to determine if the population changes under reaction conditions (e.g. if for example the $\mathrm{CO}_{2}$ were to react stoichiometrically to fill oxygen vacancies), we performed an additional control experiment where we measured the XPS spectra before and after the active hydroxide-based sample was placed in the photocatalytic reactor. As shown in Figure $\mathrm{S}_{5}$, even after 4 days of continuous reaction, no significant change was observed in the Ois shoulder peak, confirming that the population of defects does not change under reaction conditions as is expected for a catalytic material.

\section{Investigating the effect of precursor choice on sample stability under basic conditions}

Because the synthesis of the HSA-nitrate sample necessitated strongly basic etching conditions to remove the $\mathrm{SiO}_{2}$ template, it is important to establish if this treatment could have adversely affected the sample's reactivity. As a control, a sample of hydroxide-based $\mathrm{In}_{2} \mathrm{O}_{3}$ was subjected to identical etching conditions with the intent to test its photocatalytic activity and compare it to the HSA-nitrate-based sample. However, as the powder X-ray diffraction pattern in Figure $4 \mathrm{a}$ indicates, the $\operatorname{In}_{2} \mathrm{O}_{3}$ sample, which originally was comprised of pure crystalline $\mathrm{In}_{2} \mathrm{O}_{3}$ prior to etching, was converted into pure crystalline $\mathrm{In}(\mathrm{OH})_{3}$ following the etching procedure. This was quite a surprising result because the HSA-nitrate-based sample withstood this treatment very well and showed no evidence of $\operatorname{In}(\mathrm{OH})_{3}$ formation. Figure $4 \mathrm{~d}$ shows the PXRD patterns of the HSA-nitrate sample before and after base etching to remove its $\mathrm{SiO}_{2}$ template. The diffraction pattern before etching shows a broad amorphous peak around 22 degrees $2 \theta$, originating from the amorphous $\mathrm{SiO}_{2}$ nanoparticles, but the rest of the pattern is comprised of pure crystalline $\operatorname{In}_{2} \mathrm{O}_{3}$. The diffraction pattern after etching also shows pure crystalline $\operatorname{In}_{2} \mathrm{O}_{3}$ with no evidence of any other crystalline or amorphous phases. This raises two questions: (1) how does the presence of
$\mathrm{SiO}_{2}$ affect sample stability under etching conditions? and (2) is there something chemically different about the nitrate-based $\mathrm{In}_{2} \mathrm{O}_{3}$ relative to the hydroxide-based $\operatorname{In}_{2} \mathrm{O}_{3}$ ?

To answer these questions, we subjected two additional control samples to the same basic etching conditions: a pure nitrate-based $\mathrm{In}_{2} \mathrm{O}_{3}$ sample (made without $\mathrm{SiO}_{2}$ ) and a hydroxide-based sample that was combined with $\mathrm{SiO}_{2}$, dried and calcined in exactly the same way as the HSAnitrate-based sample. The result of etching the pure nitrate-based sample is shown in Figure $4 \mathrm{~b}$. While the nitrate -based $\operatorname{In}_{2} \mathrm{O}_{3}$ started out as a highly crystalline $\operatorname{In}_{2} \mathrm{O}_{3}$ sample, after etching the diffraction pattern shows a mixture of crystalline $\operatorname{In}_{2} \mathrm{O}_{3}$ and crystalline $\operatorname{In}(\mathrm{OH})_{3}$. This demonstrates that the pure nitrate-based sample is not as stable as the HSA-nitrate-based sample that was etched in the presence of $\mathrm{SiO}_{2}$. The result of etching the second hydroxide-based control sample is shown in Figure 4c. The diffraction pattern before etching is completely amorphous, despite increasing the data collection time to enhance the signal to noise ratio. It is possible that the presence of the $\mathrm{SiO}_{2}$ template inhibited the formation of the $\mathrm{In}_{2} \mathrm{O}_{3}$, though this is unlikely as the sample turned pale yellow after calcination, which is indicative of $\operatorname{In}_{2} \mathrm{O}_{3}$ formation. It is also possible that the $\operatorname{In}_{2} \mathrm{O}_{3}$ particles are so small, due to the presence of the $\mathrm{SiO}_{2}$ template that they do not diffract well enough to be seen over the amorphous background. Regardless of whether $\operatorname{In}_{2} \mathrm{O}_{3}$ was or was not formed, it is very interesting to note that, as shown in the diffraction pattern after etching, the sample does not form crystalline $\operatorname{In}(\mathrm{OH})_{3}$ under the basic etching conditions (as opposed to the hydroxide-based $\operatorname{In}_{2} \mathrm{O}_{3}$ sample made without $\mathrm{SiO}_{2}$ templating mentioned previously). Instead, this weakly crystalline pattern is almost identical to the starting diffraction pattern of the original indium hydroxide precursor (Figure S6). From these data, we can conclude that $\mathrm{SiO}_{2}$ is preferentially etched over indium-containing species and the presence of $\mathrm{SiO}_{2}$ seems to inhibit the formation of crystalline $\operatorname{In}(\mathrm{OH})_{3}$, likely by preferentially consuming hydroxide ions.

Additionally it is evident that nitrate-based samples are significantly more stable than hydroxide-based samples under these conditions. A possible explanation for this enhanced stability of nitrate-based samples under basic conditions could be explained by the differences in the thermal decomposition reactions of the indium nitrate and indium hydroxide precursors. When indium hydroxide decomposes, it releases water via Equation 1:

$$
2 \mathrm{In}(\mathrm{OH})_{3} \longrightarrow \mathrm{In}_{2} \mathrm{O}_{3}+{ }_{3} \mathrm{H}_{2} \mathrm{O}
$$

However, when indium nitrate decomposes, it releases a significantly more oxidizing gas mixture, specifically $\mathrm{NO}_{2}$ and $\mathrm{O}_{2}$, as shown in Equation 2:

$$
2 \mathrm{In}\left(\mathrm{NO}_{3}\right)_{3} \longrightarrow \mathrm{In}_{2} \mathrm{O}_{3}+6 \mathrm{NO}_{2}+{ }^{3} / 2 \mathrm{O}_{2}
$$



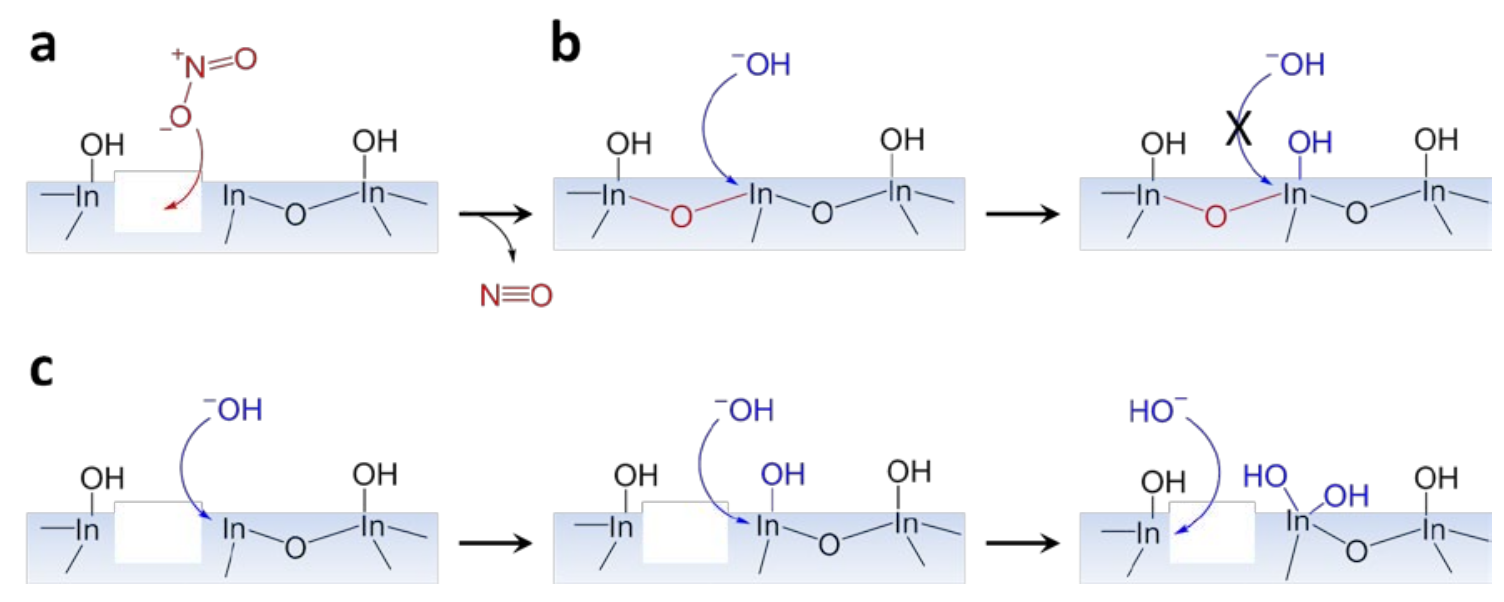

Figure 5. (a) Schematic illustration of a nitrate molecule, evolved from the decomposition of indium (III) nitrate, oxidizing a surface oxygen vacancy of the resulting indium oxide material; Schematic illustration of a hydroxide attack on (b) a hydroxylated indium oxide surface with no surface vacancies, representing the nitrate-based samples, and (c) a hydroxylated surface with oxygen vacancies, representing the hydroxide-based samples.

As the indium nitrate decomposes and begins forming $\mathrm{In}_{2} \mathrm{O}_{3}$, the $\mathrm{NO}_{2}$ that gets released can oxidize surface vacancies that are formed in the sample, as illustrated in Figure 5a. NO can easily be re-oxidized to $\mathrm{NO}_{2}$ under our reaction conditions, ${ }^{42}$ via Equation 3:

$$
\mathrm{NO}+1 / 2 \mathrm{O}_{2} \longrightarrow 2 \mathrm{NO}_{2}
$$

and continue to oxidize the surface of the nitrate-based $\mathrm{In}_{2} \mathrm{O}_{3}$ sample. Without this coordinatively unsaturated indium site, it is now much more difficult for the surface to react with hydroxide ions during the etching procedure, as illustrated in Figure 5 b. XPS data for both nitratebased samples (Figure $3 \mathrm{c}$ and $\mathrm{d}$ ) indicate a significant contribution to the O1s core level peak from oxygen vacancies, however if these vacancies are buried within the bulk of the sample, they would not be accessible to react with the evolving gases and therefore would be retained in the sample, even under these oxidizing conditions. It is unlikely that all vacancies would be consumed via this oxidation mechanism, which could explain why the nitrate-based sample (without $\mathrm{SiO}_{2}$ ) was still partially converted to $\operatorname{In}(\mathrm{OH})_{3}$ under the etching conditions.

The hydroxide-based sample on the other hand is not subjected to such oxidizing conditions during its formation and retains a significantly higher proportion of its oxygen vacancies. This makes these samples much more susceptible to attack by hydroxide ions during the etching procedure, as illustrated in Figure 5c. This could explain why neither one of the hydroxide-based $\operatorname{In}_{2} \mathrm{O}_{3}$ samples could withstand the basic etching conditions.

\section{Correlation of surface defects to photocatalytic activity}

The oxidizing nature of the nitrate-based $\operatorname{In}_{2} \mathrm{O}_{3}$ formation environment may also help to explain the significant difference in reactivity between the nitrate-based and hydroxide-based samples. Much in the same way that a coordinatively unsaturated indium site appears neces- sary to facilitate the conversion of $\operatorname{In}_{2} \mathrm{O}_{3}$ into $\operatorname{In}(\mathrm{OH})_{3}$ under basic conditions, this higher energy unsaturated site could also function as the active site for $\mathrm{CO}_{2}$ reduction. As our previous studies have demonstrated, higher defect concentrations in hydroxide-based $\mathrm{In}_{2} \mathrm{O}_{3}$ corresponded to significantly higher photocatalytic activity. ${ }^{29}$ In this study we have shown, using XPS, that both the nitrate-based and hydroxide-based samples have a significant quantity of hydroxide groups. However as our previous study suggested, surface hydroxide groups alone are not enough to explain the photocatalytic activity and instead both surface oxygen vacancies and hydroxide groups are required in an active photocatalyst. ${ }^{29,30}$ The HSA-nitrate-based sample has a comparable surface area and an even higher concentration of surface hydroxide groups than the hydroxide-based sample, however it shows no evidence of photocatalytic activity for $\mathrm{CO}_{2}$ reduction. Its stability under basic etching conditions indicates that the surface of this sample is very stable and likely lacks the coordinatively unsaturated indium sites (oxygen vacancies) needed to facilitate $\mathrm{CO}_{2}$ reduction. The hydroxide-based sample on the other hand, has both hydroxide groups as well as an increased amount of these coordinatively unsaturated indium surface sites, making it unstable under basic etching conditions and active as a photocatalyst for $\mathrm{CO}_{2}$ reduction. This stark difference in photocatalytic activity between indium oxide samples prepared from different precursors provides further evidence to support the hypothesis that a combination of surface defects - both oxygen vacancies and hydroxides - are crucial to preparing an active surface capable of driving photocatalytic reduction.

\section{Designing an active photocatalyst for $\mathrm{CO}_{2}$ reduction}

As with the synthesis of any nanomaterial, there are many parameters that can be varied that have a substantial effect on the surface, morphology, and optoelectronic properties of the resulting photocatalytic material, ultimately determining its activity. The results of this study add our increasing understanding of the necessary design parameters needed to make an active, single-component 
photocatalyst. Not only must the semiconductor nanomaterial absorb solar light efficiently, but it must also have a surface which is optimized to interact favorably with reactant molecules. In this study and in our previous work, ${ }^{29-31}$ we have demonstrated that both surface oxygen vacancies and hydroxide groups are needed to capture and convert $\mathrm{CO}_{2}$ to $\mathrm{CO}$. In order to produce an active material which contains both of these defects, carefully controlling the calcination temperature and selecting an appropriate precursor are critical. In general, lower calcination temperatures allow for the retention of more defects within the material. In the case of indium oxide, 250 ${ }^{\circ} \mathrm{C}$, which is just above the hydroxide-to-oxide transition temperature, appears to produce the most active sample, likely because it maximizes defects. In terms of precursor selection, as demonstrated herein, metal hydroxides appear to be the best choice for making hydroxylated, oxygen vacancy-rich nanomaterials. However, in general, any non-oxidizing precursor anion could be a good candidate; however one should take care when using carboncontaining anions because, as demonstrated with indium (III) acetate, substantial carbon contamination can result, complicating the analysis of the products of the photocatalytic reaction. Other parameters which we have not yet examined, but certainly would play a role in optimizing photocatalytic activity of a metal oxide nanomaterials include reaction time, atmosphere, and pressure during the synthesis of the material.

\section{Conclusions}

In this study we have demonstrated that the choice of precursor has a strong effect on the photocatalytic activity of $\operatorname{In}_{2} \mathrm{O}_{3}$ nanoparticle samples. The oxidizing nature of the precursor anion used in the nanoparticle synthesis plays a significant role in the defect formation within the sample. Precursors, such as indium nitrate, that release oxidizing gases will produce $\operatorname{In}_{2} \mathrm{O}_{3}$ nanoparticles with a reduced number of surface oxygen vacancies, rendering this material inactive as a photocatalyst. On the other hand, precursors such as indium hydroxide, which produce more mild gaseous byproducts, allow the retention of more of these surface oxygen vacancies and correlate to higher photocatalytic activities. We have further confirmed that while hydroxide groups may be important for the overall $\mathrm{CO}_{2}$ reduction reaction, simply increasing the concentration of hydroxide groups alone will not make a more active catalyst. These results demonstrate that the choice of precursor has a substantial impact on the population of surface defect sites generated during $\mathrm{In}_{2} \mathrm{O}_{3}$ synthesis, which plays an important role in determining the final photocatalytic activity. This study provides valuable insight into key parameters for the precursor selection, materials design, and performance optimization of heterogeneous gas-phase $\mathrm{CO}_{2}$ reduction photocatalysts.

\section{ASSOCIATED CONTENT}

Supporting Information. Description and characterization (powder X-ray diffraction, transmission electron microscopy, photocatalytic testing, and UV-Visible reflection spectrosco- py) of other precursors tested (indium (III) acetate and indium (III) chloride). Schematic illustration of the $\mathrm{SiO}_{2}$ templated synthesis of high surface area $\operatorname{In}_{2} \mathrm{O}_{3}$ from the indium (III) nitrate precursor. X-ray photoelectron spectroscopy (XPS) survey spectra of $\mathrm{In}_{2} \mathrm{O}_{3}$ made from hydroxide, nitrate, and high surface area nitrate. Comparison of powder X-ray diffraction patterns for $\mathrm{In}_{2} \mathrm{O}_{3}+\mathrm{SiO}_{2}$ made from indium hydroxide after the basic etching procedure and the amorphous indium hydroxide precursor used to make the hydroxidebased $\operatorname{In}_{2} \mathrm{O}_{3}$. This material is available free of charge via the Internet at http://pubs.acs.org.

\section{AUTHOR INFORMATION}

\section{Corresponding Author}

* Prof. Geoffrey A. Ozin. Department of Chemistry, University of Toronto, 8o St. George Street, Toronto, Ontario $\mathrm{M}_{5} \mathrm{~S}$ 3H6, Canada. Email: gozin@chem.utoronto.ca.

\section{Present Addresses}

${ }^{\ddagger}$ Institute of Functional Nano \& Soft Materials (FUNSOM), Jiangsu Key Laboratory for Carbon-Based Functional Materials \& Devices, Soochow University, 199 Ren'ai Road, Suzhou, 215123, Jiangsu, PR China

\section{Author Contributions}

The manuscript was written through contributions of all authors. All authors have given approval to the final version of the manuscript.

\section{ACKNOWLEDGMENT}

GAO is a Government of Canada Research Chair in Materials Chemistry and Nanochemistry. Financial support for this work was provided by the Ontario Ministry of Research Innovation (MRI); Ministry of Economic Development, Employment and Infrastructure (MEDI); Ministry of the Environment and Climate Change (MOECC); Connaught Innovation Fund; Connaught Global Challenge Fund; Natural Sciences and Engineering Research Council of Canada (NSERC). Q.Q. was supported by CCDM, an EFRC funded by the U.S. DOE-BES (\#DE-SCoo12575) for her TEM work, and Y.Z. was supported by DOE-BES, Materials Science and Engineering Division.

\section{REFERENCES}

(1) Olah, G. A.; Prakash, G. K. S.; Goeppert, A. Anthropogenic Chemical Carbon Cycle for a Sustainable Future. J. Am. Chem. Soc. 2011, 133 (33), 12881-12898.

(2) Thomas, J. M. Heterogeneous Catalysis and the Challenges of Powering the Planet, Securing Chemicals for Civilised Life, and Clean Efficient Utilization of Renewable Feedstocks. ChemSusChem 2014, 7 (7), 1801-1832.

(3) Kim, D.; Sakimoto, K. K.; Hong, D.; Yang, P. Artificial Photosynthesis for Sustainable Fuel and Chemical Production. Angew. Chemie Int. Ed. 2015, 3259-3266.

(4) Centi, G.; Quadrelli, E. A.; Perathoner, S. Catalysis for $\mathrm{CO}_{2}$ Conversion: A Key Technology for Rapid Introduction of Renewable Energy in the Value Chain of Chemical Industries. Energy Environ. Sci. 2013, 6 (6), 1711. Liu, A.; Gao, J.; He, L. New and Future Developments in Catalysis: Activation of Carbon Dioxide; Elsevier B.V., 2013. Izumi, Y. Recent Advances in the Photocatalytic Conversion of Carbon Dioxide to Fuels with Water And/or Hydrogen Using Solar Energy and beyond. Coord. Chem. Rev. 2013, 257 (1), 171-186.

(7) Zhang, N.; Ciriminna, R.; Pagliaro, M.; Xu, Y.-J. 
Nanochemistry-Derived Bi2WO6 Nanostructures: Towards Production of Sustainable Chemicals and Fuels Induced by Visible Light. Chem. Soc. Rev. 2014, 43 (15), 5276-5287.

Herron, J. A.; Kim, J.; Upadhye, A. A.; Huber, G. W.; Maravelias, C. T. A General Framework for the Assessment of Solar Fuel Technologies. Energy Environ. Sci. 2015, 8 (1), 126-157.

(9) Sternberg, A.; Bardow, A. Power-to-What? - Environmental Assessment of Energy Storage Systems. Energy Environ. Sci. 2015, 8, 389-400.

(10) Navalón, S.; Dhakshinamoorthy, A.; Alvaro, M.; Garcia, H. Photocatalytic $\mathrm{CO}_{2}$ Reduction Using Non-Titanium Metal Oxides and Sulfides. ChemSusChem 2013, 6 (4), 562-577.

(11) White, J. L.; Baruch, M. F.; Pander III, J. E.; Hu, Y.; Fortmeyer, I. C.; Park, J. E.; Zhang, T.; Liao, K.; Gu, J.; Yan, Y.; Shaw, T. W.; Abelev, E.; Bocarsly, A. B. Light-Driven Heterogeneous Reduction of Carbon Dioxide: Photocatalysts and Photoelectrodes. Chem. Rev. 2015, 115 (23), 12888-12935.

(12) Sastre, F.; Puga, A. V; Liu, L.; Corma, A.; Garcia, H. Solar Light Complete Photocatalytic $\mathrm{CO}_{2}$ Reduction to Methane by $\mathrm{H}_{2}$ at near Ambient Temperature. J. Am. Chem. Soc. 2014, 136 (19), 6798-6801.

(13) Pan, X.; Yang, M.-Q.; Fu, X.; Zhang, N.; Xu, Y.-J. Defective $\mathrm{TiO}_{2}$ with Oxygen Vacancies: Synthesis, Properties and Photocatalytic Applications. Nanoscale 2013, 5 (9), 36013614.

(14) Polarz, S.; Strunk, J.; Ischenko, V.; Van Den Berg, M. W. E.; Hinrichsen, O.; Muhler, M.; Driess, M. On the Role of Oxygen Defects in the Catalytic Performance of Zinc Oxide. Angew. Chemie - Int. Ed. 2006, 45 (18), 2965-2969.

(15) Nakamura, I.; Negishi, N.; Kutsuna, S.; Ihara, T.; Sugihara, S.; Takeuchi, K. Role of Oxygen Vacancy in the PlasmaTreated $\mathrm{TiO}_{2}$ Photocatalyst with Visible Light Activity for NO Removal. J. Mol. Catal. A Chem. 200o, 161, 205-212.

(16) Nowotny, J. Titanium Dioxide-Based Semiconductors for Solar-Driven Environmentally Friendly Applications: Impact of Point Defects on Performance. Energy Environ. Sci. 2008, 1 (5), 565-572.

(17) Liu, L.; Gao, F.; Zhao, H.; Li, Y. Tailoring $\mathrm{Cu}$ Valence and Oxygen Vacancy in $\mathrm{Cu} / \mathrm{TiO}_{2}$ Catalysts for Enhanced $\mathrm{CO}_{2}$ Photoreduction Efficiency. Appl. Catal. B Environ. 2013, 134135, 349-358.

(18) Walsh, A. Surface Oxygen Vacancy Origin of Electron Accumulation in Indium Oxide. Appl. Phys. Lett. 2011, 98 (26), $1-4$.

(19) Ye, J.; Liu, C.; Mei, D.; Ge, Q. Active Oxygen Vacancy Site for Methanol Synthesis from $\mathrm{CO}_{2}$ Hydrogenation on $\mathrm{In}_{2} \mathrm{O}_{3}$ (110): A DFT Study. ACS Catal. 2013, 3 (110), 1296-1306.

(20) Liu, G.; Hoivik, N.; Wang, K.; Jakobsen, H. Engineering $\mathrm{TiO}_{2}$ Nanomaterials for $\mathrm{CO}_{2}$ Conversion/solar Fuels. Sol. Energy Mater. Sol. Cells 2012, 105, 53-68.

(21) Lany, S.; Zakutayev, A.; Mason, T. O.; Wager, J. F.; Poeppelmeier, K. R.; Perkins, J. D.; Berry, J. J.; Ginley, D. S.; Zunger, A. Surface Origin of High Conductivities in Undoped $\mathrm{In}_{2} \mathrm{O}_{3}$ Thin Films. Phys. Rev. Lett. 2012, 108 (1), 016802.

(22) Zhang, K. H. L.; Egdell, R. G.; Offi, F.; Iacobucci, S.; Petaccia, L.; Gorovikov, S.; King, P. D. C. Microscopic Origin of Electron Accumulation in $\mathrm{In}_{2} \mathrm{O}_{3}$. Phys. Rev. Lett. 2013, 110 (5), 056803.

(23) King, P. D. C.; Veal, T. D. Conductivity in Transparent Oxide Semiconductors. J. Phys. Condens. Matter 2011, 23 (33), 334214.

(24) Habisreutinger, S. N.; Schmidt-Mende, L.; Stolarczyk, J. K. Photocatalytic Reduction of $\mathrm{CO}_{2}$ on $\mathrm{TiO}_{2}$ and Other Semiconductors. Angew. Chemie Int. Ed. 2013, 52 (29), 73727408 .

(25) Wang, G.; Wang, H.; Ling, Y.; Tang, Y.; Yang, X.; Fitzmorris, R. C.; Wang, C.; Zhang, J. Z.; Li, Y. Hydrogen-Treated TiOz Nanowire Arrays for Photoelectrochemical Water Splitting.
Nano Lett. 2011, 11 (7), 3026-3033.

(26) Ahmed, N.; Shibata, Y.; Taniguchi, T.; Izumi, Y. Photocatalytic Conversion of Carbon Dioxide into Methanol Using zinc-copper-M(III) (M=aluminum, Gallium) Layered Double Hydroxides. J. Catal. 2o11, 279 (1), 123-135.

(27) Choi, S.; Drese, J. H.; Jones, C. W. Adsorbent Materials for Carbon Dioxide Capture from Large Anthropogenic Point Sources. ChemSusChem 2009, 2 (9), 796-854.

(28) Auroux, A.; Gervasini, A. Microcalorimetric Study of the Acidity and Basicity of Metal Oxide Surfaces. J. Phys. Chem. 1990, 94 (14), 6371-6379.

(29) Hoch, L. B.; Wood, T. E.; O’Brien, P. G.; Liao, K.; Reyes, L. M.; Mims, C. A.; Ozin, G. A. The Rational Design of a Single-Component Photocatalyst for Gas-Phase $\mathrm{CO}_{2}$ Reduction Using Both UV and Visible Light. Adv. Sci. 2014, 1 (1), 1400013

(30) Ghuman, K. K.; Wood, T. E.; Hoch, L. B.; Mims, C. A.; Ozin, G. A.; Singh, C. V. Illuminating $\mathrm{CO}_{2}$ Reduction on Frustrated Lewis Pair Surfaces: Investigating the Role of Surface Hydroxides and Oxygen Vacancies on Nanocrystalline $\mathrm{In}_{2} \mathrm{O}_{3}-\mathrm{x}(\mathrm{OH}) \mathrm{y}$. Phys. Chem. Chem. Phys. 2015, 17, 14623-14635.

(31) Kaur Ghuman, K.; Hoch, L. B.; Szymanski, P.; Loh, J. Y. Y.; Kherani, N. P.; El-Sayed, M. A.; Ozin, G. A.; Veer Singh, C. Photoexcited Surface Frustrated Lewis Pairs for Heterogeneous Photocatalytic $\mathrm{CO}_{2}$ Reduction. J. Am. Chem. Soc. 2016, DOI: 10.1021/jacs.5b10179.

(32) NIST-XPS database, version 3.5. http://srdata.nist.gov/xps/.

(33) Sheets, W. C.; Mugnier, E.; Barnabé, A.; Marks, T. J.; Poeppelmeier, K. R. Hydrothermal Synthesis of DelafossiteType Oxides. Chem. Mater. 20o6, 18 (1), 7-20.

(34) Teramura, K.; Iguchi, S.; Mizuno, Y.; Shishido, T.; Tanaka, T. Photocatalytic Conversion of $\mathrm{CO}_{2}$ in Water over Layered Double Hydroxides. Angew. Chem. Int. Ed. Engl. 2012, 51 (32), 8008-8011.

(35) Yoshida, S.; Kohno, Y. A New Type of Photocatalysis Initiated by Photoexcitation of Adsorbed Carbon Dioxide on $\mathrm{ZrO} 2$. Catal. Surv. from Japan 20o1, 4 (2), 107-114.

(36) Chorkendorff, I.; Niemantsverdriet, J. W. Concepts of Modern Catalysis and Kinetics; Wiley-VCH Verlag GmbH \& Co KGaA: Weinheim, Germany, 2003.

(37) Chang, W.-C.; Kuo, C.-H.; Juan, C.-C.; Lee, P.-J.; Chueh, Y.L.; Lin, S.-J. Sn-Doped $\mathrm{In}_{2} \mathrm{O}_{3}$ Nanowires: Enhancement of Electrical Field Emission by a Selective Area Growth. Nanoscale Res. Lett. 2012, 7 (1), 684.

(38) Janowitz, C.; Scherer, V.; Mohamed, M.; Krapf, A.; Dwelk, H.; Manzke, R.; Galazka, Z.; Uecker, R.; Irmscher, K.; Fornari, R.; Michling, M.; Schmeißer, D.; Weber, J. R.; Varley, J. B.; Van de Walle, C. G. Experimental Electronic Structure of $\mathrm{In}_{2} \mathrm{O}_{3}$ and $\mathrm{Ga}_{2} \mathrm{O}_{3}$. New J. Phys. 2011, 13 (8), 085014 .

(39) Habisreutinger, S. N.; Schmidt-Mende, L.; Stolarczyk, J. K. Photocatalytic Reduction of $\mathrm{CO}_{2}$ on $\mathrm{TiO}_{2}$ and Other Semiconductors. Angew. Chemie Int. Ed. 2013, 52 (29), 73727408.

(40) Poznyak, S. K.; Golubev, A. N.; Kulak, A. I. Correlation between Surface Properties and Photocatalytic and Photoelectrochemical Activity of $\mathrm{In}_{2} \mathrm{O}_{3}$ Nanocrystalline Films and Powders. Surf. Sci. 20oo, 454-456, 396-401.

(41) Gan, J.; Lu, X.; Wu, J.; Xie, S.; Zhai, T.; Yu, M.; Zhang, Z.; Mao, Y.; Wang, S. C. I.; Shen, Y.; Tong, Y. Oxygen Vacancies Promoting Photoelectrochemical Performance of $\mathrm{In}_{2} \mathrm{O}_{3}$ Nanocubes. Sci. Rep. 2013, 3, 1021.

(42) Holleman, A. F.; Wiberg, E. Inorganic Chemistry; Academic Press: San Diego, CA, USA, 2001.

(38) Moulder, J.F.; Stickle, W.F.; Sobol, P.E.; Bomben, K. D. The Handbook of X-ray Photoelectron Spectroscopy; Eden Prairie: Physical Electronics Inc., 1995. 


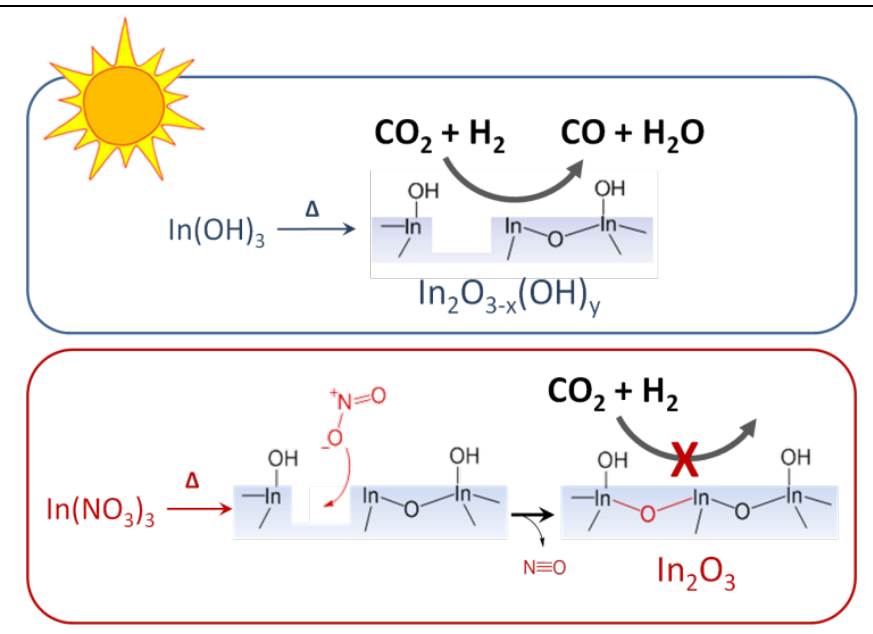

Table of Contents. Schematic illustration of the differences in surface chemistry and reactivity between indium oxide nanomaterials prepared via the thermal decomposition of either indium (III) hydroxide (top) or indium (III) nitrate (bottom). As the top box illustrates, the sample prepared from $\operatorname{In}(\mathrm{OH})_{3}$ results in the retention of surface defects, which facilitates the light driven reduction of $\mathrm{CO}_{2}$ to $\mathrm{CO}$ via the reverse water gas shift (RWGS) reaction. In contrast, as shown in the bottom box, the oxidizing gasses released during the thermal decomposition of $\operatorname{In}\left(\mathrm{NO}_{3}\right)_{3}$ result in reduced surface defect concentration and no observed activity for the RWGS reaction. 